\title{
Zinc presence in invasive ductal carcinoma of breast and its correlation with oestrogen receptor status.
}

\author{
M.J.Farquharson ${ }^{1}$, A. AlEbraheem ${ }^{2}$, K.Geraki $^{3}$, R. Leek ${ }^{4}$ and A. L. Harris ${ }^{4}$ \\ ${ }^{1}$ Department of Medical Physics and Applied Radiation Sciences, McMaster University, 1280 \\ Main St. W. Hamilton, Ontario, Canada, L8S 4L8. farquhm@ mcmaster.ca 9055259140 ext \\ 23021. \\ ${ }^{2}$ Department of Radiography, City Community and Health Sciences, City University, London, \\ EC1V 0HB, UK. Email. m.j.farquharson@ city.ac.uk, Tel 02070405694 Fax 02070405697. \\ ${ }^{3}$ Diamond Light Source, Harwell Science and Innovation Campus, Didcot, Oxon, OX11 ODE, \\ UK \\ ${ }^{4}$ Cancer Research UK, Oxford Cancer Centre, Molecular Oncology Laboratories, University of \\ Oxford, Weatherall Institute of Molecular Medicine, John Radcliffe Hospital, Oxford, 0X3 9DS, \\ UK
}

\section{PACS category}

87.16.-b

\section{Short title}

Zinc concentrations and ER status in breast cancer

\section{Keywords}

X-ray fluorescence, Breast Cancer, Oestrogen receptor status, Trace element mapping, Synchrotron radiation.

\begin{abstract}
Zinc is known to play an important role in many cellular processes and the levels of zinc are controlled by specific transporters from the ZIP (SLC39A) influx transporter group and the ZnT (SLC30A) efflux transporter group. The distribution of zinc was measured in samples of invasive ductal carcinoma of breast using synchrotron radiation micro probe $\mathrm{x}$-ray fluorescence facilities. The samples were formalin fixed paraffin embedded Tissue Micro Arrays (TMAs) enabling a high throughput of samples and allowing us to correlate the distribution of trace metals with tumour cell distribution and, for the first time, important biological variables. The samples were divided into two classes, oestrogen receptor positive $(E R+v e)$ and oestrogen receptor negative
\end{abstract}


(ER-ve) based on quantitative immunohistochemistry assessment. The overall levels of zinc (i.e. in tumour and surrounding tissue) in the ER+ve samples was on average $60 \%$ higher than in the ER-ve samples. The zinc levels were higher in the ER+ve tumour areas compared to the ER-ve tumour areas with the mean levels in the ER+ve samples being approximately $80 \%$ higher than the mean ER-ve levels. However, the non tumour tissue regions of the samples contained on average the same levels of zinc in both types of breast cancer. The relative levels of zinc in tumour areas of the tissue were compared with levels in areas of non tumour surrounding tissue. There was a significant increase in zinc in the tumour regions of the ER+ve samples compared to the surrounding regions $(\mathrm{P}<0.001)$ and a non significant increase in the ER-ve samples. When comparing the increase in zinc in the tumour regions expressed as a percentage of the surrounding non tumour tissue zinc level in the same sample, a significant difference between the ER+ve and ER-ve samples was found $(\mathrm{P}<0.01)$.

\section{Introduction}

Zinc is an essential element for life and has been established as being of great importance in humans. It is an essential ion in cells and is a cofactor for more than 300 enzymes. Zinc deficiency can have detrimental effects in humans e.g. in acrodermatitis enteropathica, an inherited defect in which zinc absorption from the intestine is reduced resulting in retarded growth, hypogonadism, skin \& ophthalmic lesions, and a deficiency in cell-mediated immunity. However, an excess of zinc can be toxic to cells, hence the regulation of cellular zinc levels is of great importance.

The concentrations of zinc in normal and diseased tissue from a number of organs have been measured. Reddy et al (2008), used particle induced x-ray emission (PIXE) techniques to carry out trace element analysis on samples of kidney and stomach carcinoma. The study reported higher levels of zinc in the cancerous tissue of the kidney compared to normal tissue, while in the stomach the zinc levels were reported to be lower in the cancerous tissue. Kwiatek et al (2002) also investigated trace element levels in cancerous kidney by using Synchrotron Radiation Induced X-ray Emission (SRIXE), reporting a lower concentration of zinc in the cancerous liver when compared to normal tissue. AlEbraheem et al (2009) measured concentrations of zinc in both kidney and liver tumours and compared them with normal kidney and liver tissue using $\mathrm{x}$ ray fluorescence. The study reported that zinc was reduced in liver and kidney tumours by $63 \%$ 
and 26\%, respectively. Tashiro et al (2006) studied levels of zinc in the liver and reported lower levels in the tumour tissue compared to non tumour tissue. Carvalho and Marques (2001) also looked at liver tissue using x-ray fluorescence and reported lower values of $\mathrm{Zn}$ in liver cirrhosis samples compared with healthy tissues. Work by our group (Farquharson et al 2008) carried out $\mathrm{XRF}$ analysis on secondary colorectal liver metastases and reported a significant lowering of zinc levels $(\mathrm{p}<0.01)$ in the cancer tissue when compared to the normal surrounding liver tissue. The role of zinc in prostate cancer has been of interest in recent years and there have been a number of studies looking at the concentrations in cancer and normal prostate tissue. IdeEktessabi et al (2002) used a synchrotron X-ray fluorescence microbeam to measure levels of zinc in cancerous and normal tissues of the human prostate. In the cancerous tissues, zinc was reported at levels of $89-221 \mathrm{ppm}$ in the adenocarcinoma and 44-713 ppm in the stroma. In the normal tissues, zinc was detected at 158-474 ppm in the epithelial cells and 101-180 ppm in the stroma.

In the case of breast cancer there have been a number of studies looking at trace element levels. Garg et al (1990) used neutron activation analysis (INAA) to study breast tumours and healthy breast tissue and reported elevated levels of all measured elements in the tumour samples including $\mathrm{Fe}, \mathrm{Cu}$ and $\mathrm{Zn}$. Ng et al (1997) also used INAA in a similar study on both healthy and cancerous breast tissue and reported all measured elements increased in the tumour tissue again including $\mathrm{Fe}, \mathrm{Cu}$ and $\mathrm{Zn}$. Total reflection XRF was utilized by Majewska et al (1997) to compare benign and malignant breast tumours and reported increased levels of most elements measured including $\mathrm{K}, \mathrm{Ca}, \mathrm{Fe}, \mathrm{Cu}, \mathrm{Zn}, \mathrm{Se}$ and $\mathrm{Rb}$. The same technique was used by Kubala-Kukus et al (2007) in a study that compared malignant and benign tumours in breast and reported higher levels of all elements measured in malignant tumours with the exception of $\mathrm{Cu}$ which was found to be reduced. Majewska et al (2007) carried out a very similar study again using total reflection $\mathrm{XRF}$ and reported significant higher levels of Fe and Se in malignant breast tissue compared to benign tissue. Geraki et al $(2002,2004)$ used synchrotron radiation to evaluate the levels of $\mathrm{K}$, $\mathrm{Fe}, \mathrm{Cu}$ and $\mathrm{Zn}$ in 40 matched pairs of normal breast tissue and tumour tissue reporting significant higher levels of all elements in the cancer samples. A recent paper by Silva et al (2008) has reported elevated levels of $\mathrm{Ca}, \mathrm{Fe}, \mathrm{Cu}$ and $\mathrm{Zn}$ in tumour breast tissue compared to healthy tissue from the same breast. More recent work by our group has made use of synchrotron radiation $\mathrm{x}$ ray fluorescence (SRXRF) micro probes to map the distribution of metals including zinc in 
samples of invasive ductal carcinoma of breast (Farquharson et al 2008, Geraki et al 2007). The advantage of mapping the distribution of metals in the tissue compared to measuring levels in a lump of tissue can be seen by example when comparing the results presented for iron. Our work on iron levels in breast cancer using a lump of tissue have reported higher levels in the tumour compared to healthy tissue. On average this appears to be the case but our mapping technique has shown that there are some cases where the iron levels are decreased in the tumour compared to surrounding normal tissues and seems in some way to be case specific.

Cellular concentrations of zinc are strictly controlled by zinc transporter proteins that fall into two families. One is the ZnT family (SLC30A) that are responsible for the control of zinc efflux, transporting zinc out of the cells or into intracellular compartments. The human $\mathrm{ZnT}$ fimily contains 9 genes. The second is the ZIP family (SLC39A) that control the zinc influx, transporting zinc into the cell cytoplasm from outside the cell or from intracellular regions. The human ZIP family has 14 genes. Furthermore, it has been reported that certain members of the ZIP transporter family play a role in various disease states suggesting that an uncontrolled expression of zinc transporters could lead to an uncontrolled growth such as occurs in cancer (Taylor et al 2007). There is one sub group of the ZIP family that contains 9 genes and is known as the LIV-1 subfamily. Eight of these are transmembrane proteins residing on the plasma membrane of cells and transport zinc into the cell, but one member is situated on intracellular membranes resulting in it transporting zinc from intracellular compartments to the cytoplasm.

Oestrogen is a primary female sex hormone that acts by binding and activating protein molecules called oestrogen receptors (ER). Oestrogen is important for the normal growth and development of the mammary gland and breast cancer progression is influenced by oestrogen, (Anderson 2002). An assessment of ER expression within the tumour cells can be stated to be ER negative when the tumour does not show ER expression, or ER positive when the tumour shows expression of ER (Badve and Nakshatri 2009). ZIP7 has been shown to be regulated by oestrogen and has elevated expression in oestrogen receptor positive (ER+ve) cancer of the breast and an inverse relationship to epidermal growth factor receptor (EGFR) status (Taylor et al 2007). Given its role as a regulator of zinc influx, the increased levels of LIV-1 could lead to a higher concentration of zinc in ER+ve cancers compared to ER-ve cancers. We therefore 
investigated the zinc levels in human primary tumour tissue compared to surrounding stromal tissue in samples of both ER + ve and ER - ve invasive ductal carcinoma of breast. In addition to using ER status as a marker, we also compared the levels using EGFR status, nodal status, tumour size, tumour grade and menopausal status.

\section{Methodology}

The data was collected using Synchrotron Radiation X-Ray Fluorescence (SRXRF) microprobes at three locations. 1. The FLUO beamline at ANKA, Karlsruhe, Germany. 2. Beamline L, Hasylab, DESY, Hamburg and beamline I18 at DIAMOND in the UK. The experimental set up took on a similar form at all three facilities with specific details given below. At DESY the data was collected using the Synchrotron X-Ray Fluorescence Microprobe at Hasylab, beamline L The bending magnet source produces a white beam that is monochromated by a double multilayer monochromator. The beam is focussed by a polycapillary half lens (X-Ray Optical Systems, Inc). At the energy of $11 \mathrm{keV}$ used at DESY for this study an on sample spot size of approximately $15 \mu \mathrm{m} \times 15 \mu \mathrm{m}$ was obtained. A stepwise scan was used with the measurement time at each point being 5 seconds. The fluorescence signal is recorded using a Peltier cooled energy dispersive Si drift detector (Radiant, Vortex). The data is further analysed using AXIL, a programme that fits the element $K_{\alpha}$ and $K_{\beta}$ peaks under consideration taking into account line overlaps and subtracts background. In this case the data is normalised to the varying incident radiation intensity via an ionisation chamber measurement pre sample.

The data collected at ANKA using the FLUO beamline which again has a bending magnet source monochromated using double multilayer monochromator. A poly capillary lens gives an on sample spot size of approximately $3 \mu \mathrm{m}$ x $5 \mu \mathrm{m}$ at an energy of $10.5 \mathrm{keV}$. The data is collected using a $\mathrm{Si}(\mathrm{Li})$ energy dispersive detector (Oxford Instruments) and processed using AXIL. Here the data collection time is allowed to vary according to the length of time for a pre set amount of radiation to reach the sample at each measurement point.

The undulator-produced beam on I18 at Diamond is focused by a pair of Kirkpatrick-Baez mirrors after being monochromatised by a double-crystal monochromator. On this occasion the on sample size of the beam was approximately $5 \mu \mathrm{m} \times 5 \mu \mathrm{m}$. A 9-element Ortec germanium detector was used while the treatment of the spectra was accomplished using PyMCA (Solé et al 2007). The incident beam energy was $11 \mathrm{keV}$ and the collection time per measurement point was 
4 seconds. Note this facility is constant top up and normalisation to beam intensity was not required as variation was negligable.

At all facilities a $45^{\circ}$ geometry was used between the incident radiation beam and the sample, and a $90^{\circ}$ geometry between the incident beam and the detector. A schematic of the experimental set up is shown in figure 1. A novel feature of this work was that the samples were in the form of formalin fixed paraffin embedded Tissue Micro Arrays (TMAs) consisting of $1.0 \mathrm{~mm}$ diameter sections of tissue mounted in arrays of size $5 \times 4$ samples. Up to six of these arrays are mounted on any one slide. The samples were pre-screened in order to identify those suitable for this study, i.e. that they were invasive ductal carcinoma (IDC) and the ER status identified so that as near equal numbers of each classification could be used. Two slices were cut from the paraffin block, one being $10 \mu \mathrm{m}$ thick the other being $5 \mu \mathrm{m}$ thick and cut adjacent to the $10 \mu \mathrm{m}$ slice. The $10 \mu \mathrm{m}$ thick slice was mounted on $4 \mu \mathrm{m}$ ultralene XRF film that was stretched and held in a bespoke frame keeping the sample slice as flat as possible. This is important in that it enabled the sample to be kept in a constant plane with respect to the incident $\mathrm{x}$-ray beam. The $5 \mu \mathrm{m}$ thick slice was mounted on a standard glass slide and then stained using Haematoxylin and Eosin ( $\mathrm{H} \& \mathrm{E})$. This slide was then optically imaged to produce high resolution images which clearly identify tumour regions in the samples and can be used as a reference slide for the elemental distribution maps produced from the experimental slide. All samples were supplied by the Cancer Research UK Tumour Pathology Group, University of Oxford, Nuffied, Department of Clinical Laboratory Sciences, John Radcliffe Hospital, Oxford UK. 
$1 \mathrm{~mm}$ dia. Sample with region of interest shown

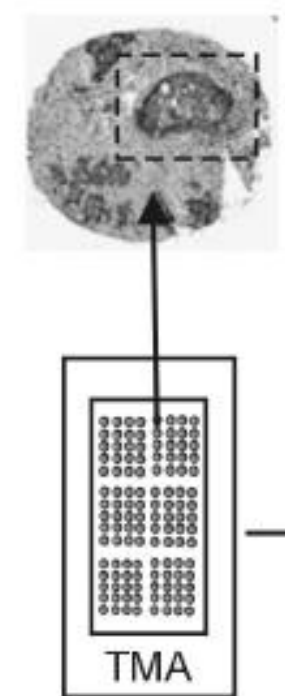

Tissue Micro Array

Spectra at each position

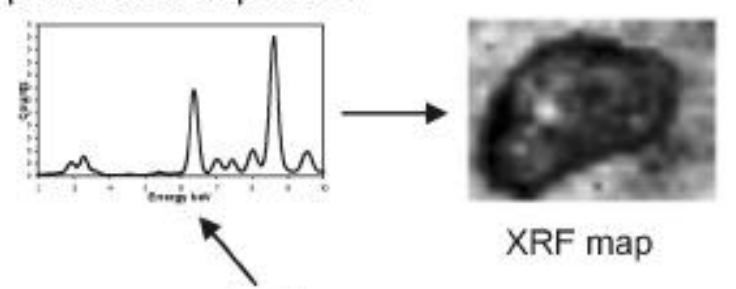

Scattered beam and XRF response

Silicon Drift or Ge detector

Figure 1: Schematic showing samples arranged as a tissue micro array and mounted on a sample holder held on an $x y z$ translator stage. The sample array is positioned at $45^{\circ}$ to the synchrotron beam. The detector is at $90^{\circ}$ to the beam. At each measurement point the $K_{\alpha} X R F$ peaks are fitted and the areas calculated resulting in a distribution map of each element.

Maps of the zinc distribution were produced and could be compared to the $\mathrm{H}$ and $\mathrm{E}$ reference images, which enabled regions of interest to be set on the maps encompassing tumour cell regions and regions of surrounding non tumour tissue. Each pixel represents a value that was the total zinc $\mathrm{K}_{\mathrm{a}}$ count normalised for the intensity of the incident beam. Statistics could then be extracted from these regions including mean normalised pixel value, maximum and minimum normalised pixel value and the standard deviation. Figure 2 shows a map of the zinc distribution compared to the stained reference image with the region of interests set for this particular sample. 


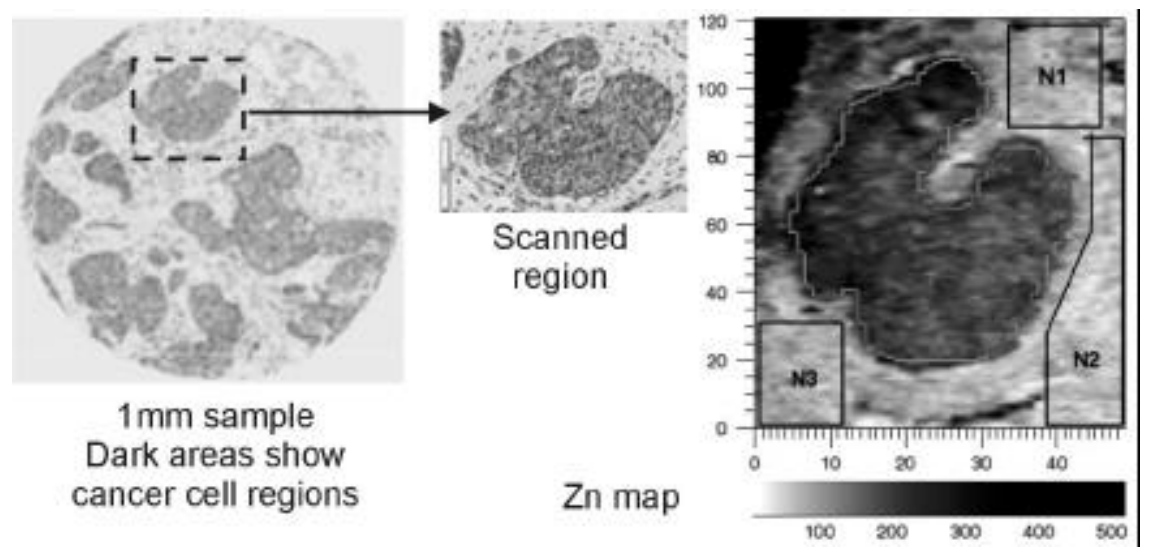

Figure 2 : A zinc map of a region of one sample with a spatial resolution of approximately $2.5 \mu \mathrm{m} \times 5 \mu \mathrm{m}$. The dark area is outlined as a tumour region of interest and in this case three regions are set for non tumour tissue.

(Data obtained using the FLUO beam line, ANKA)

\section{Results}

A total of 34 ER+ve and 25 ER-ve samples were scanned and analyzed. Figure 3 shows a section of one of the samples that was scanned at the Fluo beam line, ANKA. The scan size is $250 \mu \mathrm{m} \mathrm{x}$ $250 \mu \mathrm{m}$ with an on sample beam size of $5 \mu \mathrm{m}$ along the $\mathrm{x}$ axis and $2.5 \mu \mathrm{m}$ along the $\mathrm{y}$ axis giving a matrix of $50 \times 100$ pixels. The sample is invasive ductal carcinoma of grade 1, the ER status is positive and the EGFR status negative. The dark areas of the reference image show the tumour areas and the zinc distribution map is also shown. A line profile is shown giving an indication of the difference in zinc concentrations across tumour and non tumour tissue. It can also be seen that the response from the XRF ultralene film on which the samples are mounted is very small. Also shown are the regions of interest set from which statistical data can be acquired. In this particular example the mean normal tissue pixel value is 100.5 normalised counts while the mean tumour pixel value is 347 . The percentage increase in the zinc in tumour regions with respect to the surrounding non tumour regions is approximately $246 \%$. 

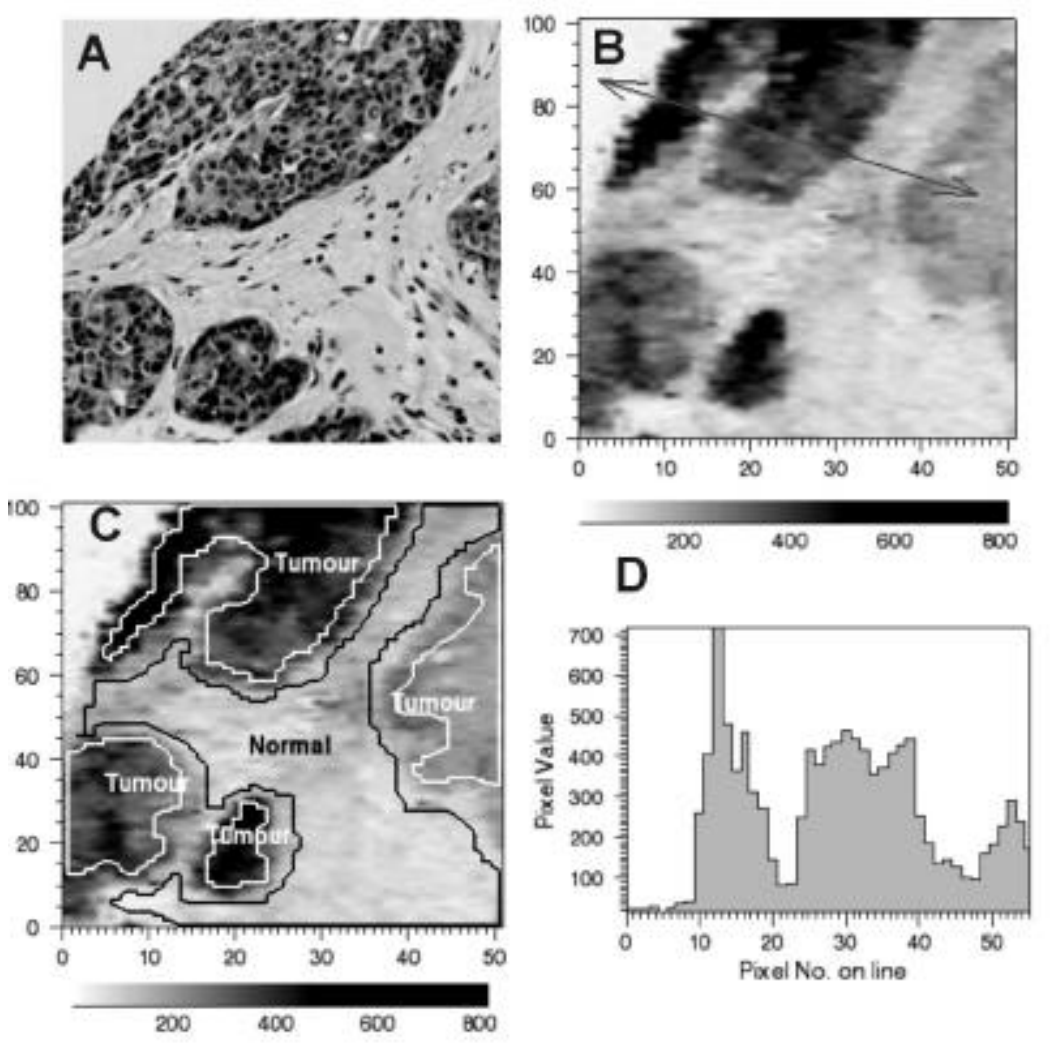

Figure 3: An example of a scan with $\boldsymbol{A}$ showing the reference $H$ and $E$ stained image and $\boldsymbol{B}$ the corresponding zinc distribution map. The line on figure $\boldsymbol{B}$ is the profile line corresponding to figure $\boldsymbol{D}$ showing a histogram of pixel intensities or variations in zinc levels along the line. Figure $\boldsymbol{C}$ shows how the regions of interest were set on this sample. (Data obtained using the FLUO beam line, ANKA)

When comparing the zinc levels between the ER+ve and the ER-ve groups it was found that the mean total zinc content for each sample (i.e. tumour and surrounding regions) was approximately $60 \%$ higher in the ER+ve samples than the ER-ve samples. There was also difference in the mean levels of zinc in the tumour regions with ER+ve samples having approximately $80 \%$ more zinc than ER-ve samples. The mean levels of zinc in the non tumour regions did not differ significantly although on average the increase in the ER+ve samples was approximately $10 \%$ more than the ER-ve samples. The zinc levels in the tumour and surrounding regions of the ER+ve samples were compared. For all of the samples, the tumour tissue zinc levels were higher than the corresponding surrounding non tumour tissue zinc, the difference being very significant $(\mathrm{p}<0.001 \mathrm{t}$-test). For the ER-ve samples, the zinc levels were higher in the tumour tissue for all samples, however the analysis revealed that the difference was not significant $(\mathrm{P}=0.07)$. Figure 4 shows the correlation between the levels of zinc in the non tumour tissue and tumour tissue 
regions in the samples. There is a similar correlation in the ER+ve and ER-ve samples (Pearson product moments correlation squared, $\mathrm{R}^{2}=0.35$ and 0.38 respectively).

The average values for the percentage increase in zinc in tumour regions with respect to surrounding regions for the ER+ve samples was 148\%, while for the ER-ve samples was $89 \%$. Table 1 shows a summary of the analyses for both sets of data. The higher percentage increase in zinc values in the ER+ve samples compared to the ER-ve samples was significantly different $(\mathrm{P}<0.01)$.
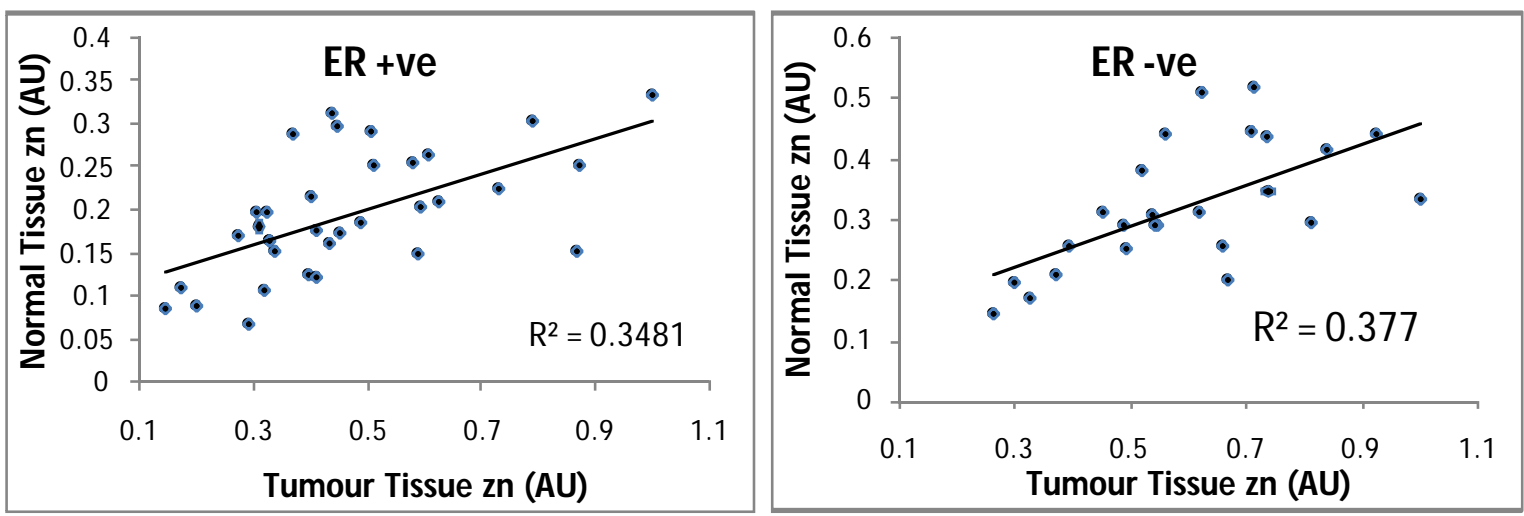

Figure 4: Correlation between the levels of zinc in the normal tissue and tumour tissue regions in the ER+ve and ER-ve samples. Note the data has been normalised to the highest value in each set.

\begin{tabular}{|c|c|c|}
\hline \multirow{2}{*}{} & \multicolumn{2}{|c|}{$\begin{array}{c}\text { Percentage increase of zn in tumour tissue with } \\
\text { respect to normal tissue }\end{array}$} \\
\cline { 2 - 3 } & $\mathrm{ER}+\mathrm{ve}$ & $\mathrm{ER}-\mathrm{ve}$ \\
\hline Mean & 148 & 89 \\
\hline SD & 95 & 53 \\
\hline Max & 471 & 233 \\
\hline Min & 27 & 21 \\
\hline Range & 444 & 211 \\
\hline $\begin{array}{c}\text { Difference in zn levels } \\
\text { between normal and } \\
\text { tumour tissue }\end{array}$ & $\mathrm{P}<0.001$ & $\mathrm{P}=0.7$ \\
\hline
\end{tabular}

Table 1: Statistical summary of the percentage difference in zinc levels in tumour with respect to the normal liver tissue. 
The range of values for both sets of data is large with the standard deviation as a percentage of the mean being $64 \%$ for the ER+ve data and $58 \%$ for the ER-ve data. Figure 5 shows a frequency distribution for both sets of data. The lower values of the differences in zinc levels are predominantly ER-ve samples and the higher values ER+ve samples. However, the large range and overlap of data sets is clear.

The samples were reclassified in terms of their EGFR status, nodal status, tumour size, tumour grade and menopausal status. A summary of the results are shown in table 2.

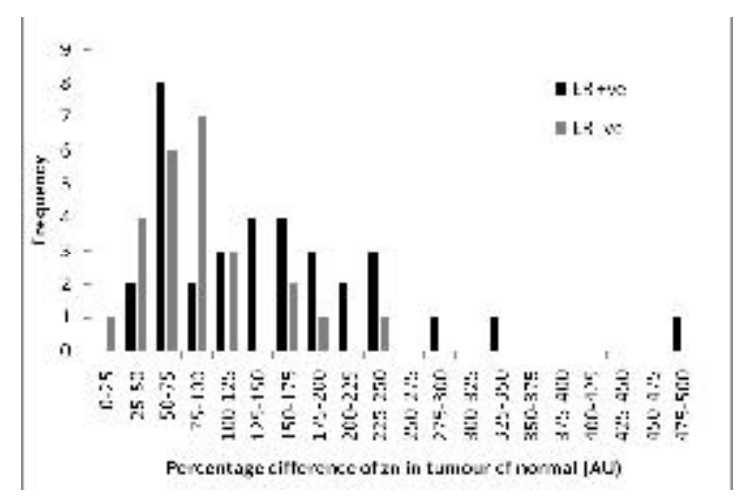

Figure $5:$ Frequency distribution for both sets of data. The precentage difference of zinc in tumour with respect to normal tissue is binned in units of $25 \%$.

The EGFR, nodal and menopausal markers showed no significant differences between status. Beside the ER status result, the only significant results were found in the tumour size and tumour grade. The size of tumour was split into three groups, $0-1.5 \mathrm{~cm}, 1.5-3.0 \mathrm{~cm}$ and greater than $3.0 \mathrm{~cm}$. The significant difference was found between the samples in grouping $0-1.5$ and the samples in grouping 1.5-3.0. The tumour grade was split into grades 1, 2 and 3 with a significant difference found between tumours with grades 1 and 2 . 


\begin{tabular}{|c|c|c|c|}
\hline Marker & Sample size (n) & $\begin{array}{c}\text { Mean \% } \\
\text { difference }\end{array}$ & $\begin{array}{c}\text { Significance } \\
\text { t test }\end{array}$ \\
\hline \multicolumn{4}{|l|}{ ER status } \\
\hline+ ve & 34 & 148 & \multirow{2}{*}{$S p=0.034$} \\
\hline -ve & 25 & 89 & \\
\hline \multicolumn{4}{|c|}{ EGFR status } \\
\hline$+\mathrm{ve}$ & 29 & 115 & \multirow{2}{*}{ NS $p=0.48$} \\
\hline -ve & 24 & 114 & \\
\hline \multicolumn{4}{|c|}{ Nodal status } \\
\hline$+\mathrm{ve}$ & 24 & 114 & \multirow{2}{*}{ NS $p=0.67$} \\
\hline -ve & 23 & 123 & \\
\hline \multicolumn{4}{|c|}{ Tumour size } \\
\hline $0-1.5$ & 12 & 143 & \multirow{2}{*}{$S p=0.029$} \\
\hline $1.5-3.0$ & 28 & 100 & \\
\hline $1.5-3.0$ & 28 & 100 & \multirow{2}{*}{ NS $p=0.49$} \\
\hline$>3.0$ & 12 & 115 & \\
\hline \multicolumn{4}{|c|}{ Tumour grade } \\
\hline 1 & 8 & 153 & \multirow{2}{*}{$S p=0.043$} \\
\hline 2 & 17 & 101 & \\
\hline 2 & 17 & 101 & \multirow{2}{*}{ NS $p=0.97$} \\
\hline 3 & 27 & 100 & \\
\hline \multicolumn{4}{|l|}{ Menopause } \\
\hline Post & 42 & 108 & \multirow{2}{*}{ NS $p=0.22$} \\
\hline Pre & 12 & 135 & \\
\hline
\end{tabular}

Table 2: A summary of the results for comparisons in the percentage difference in zinc levels between tumour and surrounding tissue for EGFR status, nodal status, tumour size, tumour grade and menopausal status.

\section{Conclusion}

To the best of our knowledge, this is the first study to map the distribution of zinc and be able to correlate the findings with important biological variables. The technique has the advantage of being able to discriminate the metal levels between tumour tissue and the surrounding stroma tissue. The increase in zinc levels in ER+ve invasive ductal carcinoma using a micro-mapping technique has not been previously described. A likely explanation of these data is related to the 
elevated levels of the zinc transporter family reported in ER+ve cancers. These transporters exist on the plasma membrane and the higher levels result in an increase influx of zinc into the tumour cells. The observation that the zinc levels in the normal regions of both ER+ve and ER-ve were approximately the same would suggest that there is an uninterrupted supply of zinc to the tissue. However, if there is an inverse relationship for the zinc transporter, ZIP7 with EGFR status i.e. lower amounts of zinc transporter in EGFR+ve cancers it could be expected that zinc concentrations may be lower in these cancers compared to EGFR-ve cancers. However this study found no difference in zinc levels between these groups, a possible explanation being that zinc is transported to the tumour cells using an alternative transport pathway. It should be noted that some zinc transporters on the plasma membrane do not show this inverse relationship i.e. ZIP5, ZIP10 and ZIP14 (Taylor et al 2007), and so may provide a way of supplying zinc.

An uptake of zinc could be clinically relevant and this study shows the feasibility of assessing this in large numbers of small samples. Antihormone drugs such as tamoxifen and aromatase inhibitors are used to treat ER+ve breast cancers but the tumours often develop resistance to these drugs. In some cases regrowth of the tumour and a progression in the disease is via the activation of members of the EGFR family such as HER2. It has been proposed that inhibition of the zinc transporter reduces activation of the EGFR signaling by reducing zinc levels and therefore offers way of preventing further development of the cancer (Taylor et al 2008). Zinc chelating agents are a possible treatment as they have been shown to exhibit antitumor effects in human breast cancer by inducing apoptosis (Hashemi et al 2007). However, these chelators may result in significant cytotoxic effects. These toxic effects may also be via inhibition of the antioxidant induction of superoxide dismutase (SOD). It is clear it would be desirable to select the subgroup of patients with high tumour zn levels for such trials, rather than treat all patients. This method and results show for the first time the relationship of $\mathrm{zn}$ in breast tumours to breast tumour biology and opens the way for further investigations of the mechanism of zinc uptake in clinical samples and assays to select and monitor zn chelation therapy. It is clear that zinc plays an important role in the development of breast and possibly other cancers and is an element that warrants further study.

\section{Acknowledgements}

This work was performed using the FLUO beamline at ANKA, Karlsruhe, Germany, Beamline 
L, Hasylab, DESY, Hamburg, Germany and Beamline I18 at Diamond, The authors are grateful to the beam line team of R. Simon (ANKA), K. Rickers (DESY) and F. Mosselmans and P. Quinn at Diamond. We would also like to acknowledge Cancer Research UK for funding A.L.Harris and R.Leek.

\section{References.}

Al-Ebraheem. A, Farquharson M.J. and Ryan E. 2009 The evaluation of biologically important trace metals in liver, kidney and breast tissue. Applied Radiation and Isotopes. 67 470-474

Anderson E 2002 Progesterone receptors - animal models and cell signaling in breast cancer: The role of oestrogen and progesterone receptors in human mammary development and tumorigenesis Breast Cancer Research 4 197-201

Badve S and Nakshatri H 2009 Oestrogen-receptor-positive breast cancer: towards bridging histopathological and molecular classifications J Clin Pathol 62 6-12

Carvalho M L and Marques A F 2001 X-ray fluorescence spectrometry: applications in trace elements studies in human tissues from patients with cirrhosis X-Ray Spectrometry 30 397-402

Farquharson M.J., Al-Ebraheem A., Theodorakou C., Ryan E., Bradley D., Gurasamy K and Davidson B. 2008 Trace elements in secondary colorectal liver cancer and their use as a tissue classifier. X-Ray Spect. DOI (DOI: 10.1002 /xrs.1121).

Farquharson M.J., Al-Ebraheem A., Falkenberg G., Leek R., Harris A.L. and Bradley D.A. 2008 The distribution of trace elements $\mathrm{Ca}, \mathrm{Fe}, \mathrm{Cu}$ and $\mathrm{Zn}$ and the determination of copper oxidation state in breast tumour tissue using $\mu$ SRXRF and $\mu$ XANES. Phys. Med Biol. 53 3023- 3037

Garg A N, Weginwar R G, and Sagdeo V 1990 Minor and Trace Elemental Contents of Cancerous Breast-Tissue Measured by Instrumental and Radiochemical Neutron-Activation Analysis Biological Trace Element Research 26-27 485-496 
Geraki K. Farquharson M.J. and Bradley 2002 Concentrations of $\mathrm{Fe}, \mathrm{Cu}$ and $\mathrm{Zn}$ in breast tissue, a synchrotron study. Phys. Med. Biol. 47(13) 2327-2339

Geraki K, Farquharson M.J. and Bradley D.A. 2004 X-ray fluorscence and energy dispersive X-ray diffraction for the quantification of elemental concentrations in breast tissue. Phys. Med Biol 49 (1) 99-110

Geraki K, Farquharson M.J., Bradley D.A. Gundogdu O and Falkenberg G. 2007 The localisation of biologically important elemental metals in soft and calcified tissues using a synchrotron x-ray fluorescence technique. $x$-ray spectrometry. 37(1) 12-20

Hashimi M., Ghavami S., Eshraghi M., Booy E.P. and Los M. 2007 Cytotoxic effects of intra and extracellular zinc chelation on human breast cancer cells. European Journal of Pharmacology. 557 9-19

Ide-Ektessabi A, Fujisawa S, Sugimura K, Kitamura Y, and Gotoh A 2002 Quantitative analysis of zinc in prostate cancer tissues using synchrotron radiation microbeams X-Ray Spectrometry 31 7-11

Kubala-Kukus A, Banas D., Braziewicz j., Gózdz S., Majewska U. and Pajek M. 2007 Analysis of elemental concentration censored distributions in breast malignant and breast benign neoplasm tissues. Spectrochimica Acta Part B 62 695-701

Kwiatek W M, Drewniak T, Gajda M, Galka M, Hanson A L, and Cichocki T 2002 Preliminary study on the distribution of selected elements in cancerous and non-cancerous kidney tissues Journal of Trace Elements in Medicine and Biology 16 155-160

Majewska U, Braziewicz J, Banas D, Kubala-Kukus A, Gozdz S, Pajek M, Smok J and Urbaniak A 1997 An elemental correlation study in cancerous breast tissue by total reflection x-ray fluorescence Biol. Trace Elem. Res. 60 91-100 
Majewska U., Bana’s D., Braziewicz J, Stanislaw G, Kubala-Kuku A. and Kucharzewski M. 2007 Trace element concentration distributions in breast, lung and colon tissues Phys. Med. Biol. 52 3895-3911

Ng K H, Bradley D A, and Looi L M 1997 Elevated trace element concentrations in malignant breast tissues British Journal of Radiology 70 375-382

Reddy S B, Charles A J, Raju G J N, Vijayan V, Reddy B S, Kumar M R, and Sundareswar B 2003 Trace elemental analysis of carcinoma kidney and stomach by PIXE method Nuclear Instruments \& Methods in Physics Research Section B-Beam Interactions with Materials and Atoms 207 345-355

Silva M.P., Tomal A., Perez C.A., Ribeiro-Silva A. and Poletti M.E. 2008 Determination of Ca, $\mathrm{Fe}, \mathrm{Cu}$ and $\mathrm{Zn}$ and their correlations in breast cancer and normal adjacent tissues. X-Ray Spectrometry DOI 10.1002/xrs.1126

Tashiro H, Kawamoto T, Okubo T, and Koide O 2003 Variation in the distribution of trace elements in hepatoma Biological Trace Element Research 95 49-63

Taylor K.M., Vichova P., Jordan N., Hiscox S., Hendley R. and Nicholson R.I. 2008 ZIP 7Mediated intracellular zinc transport contributes to aberrant growth factor signalling in antihormone resistant breast cancer cells. Endocrinology 149(10) 4912-4920

Taylor K.M., Moragan H.E., Smart K., Zahari N.M., Pumford S., Ellis I.O., Robertson J.F.R. and Nicholson R.I. 2007 The emerging role of the LIV-1 subfamily of zinc transporters in breast cancer. Mol. Med. 13(7-8) 396-406

V.A. Solé, E. Papillon, M. Cotte, Ph. Walter, J. Susini, A multiplatform code for the analysis of energy-dispersive X-ray fluorescence spectra, Spectrochim. Acta Part B 62 (2007) 63-68. 